PROCEEDINGS OF THE

AMERICAN MATHEMATICAL SOCIETY

Volume 128, Number 11, Pages 3411-3419

S 0002-9939(00)05573-8

Article electronically published on May 18, 2000

\title{
CONVERGENCE OF PATHS FOR PSEUDO-CONTRACTIVE MAPPINGS IN BANACH SPACES
}

\author{
CLAUDIO H. MORALES AND JONG SOO JUNG
}

(Communicated by David R. Larson)

\begin{abstract}
Let $X$ be a real Banach space, let $K$ be a closed convex subset of $X$, and let $T$, from $K$ into $X$, be a pseudo-contractive mapping (i.e. $(\lambda-1)$ $\|u-v\| \leq\|(\lambda I-T)(u)-(\lambda I-T)(v)\|$ for all $u, v \in K$ and $\lambda>1)$. Suppose the space $X$ has a uniformly Gâteaux differentiable norm, such that every closed bounded convex subset of $K$ enjoys the Fixed Point Property for nonexpansive self-mappings. Then the path $t \rightarrow x_{t} \in K, t \in[0,1)$, defined by the equation $x_{t}=t T x_{t}+(1-t) x_{0}$ is continuous and strongly converges to a fixed point of $T$ as $t \rightarrow 1^{-}$, provided that $T$ satisfies the weakly inward condition.
\end{abstract}

\section{INTRODUCTION}

Let $X$ be a real Banach space and $D$ a subset of $X$. An operator $T: D \rightarrow X$ is said to be pseudo-contractive (see [4, 16]) if for each $u$ and $v$ in $D$ and $\lambda>1$

$$
(\lambda-1)\|u-v\| \leq\|(\lambda I-T)(u)-(\lambda I-T)(v)\|
$$

(with $I$ denoting the identity mapping). Following Kato [12], we find an equivalent formulation of (11) that can be described as follows: a mapping $T: D \rightarrow X$ is pseudo-contractive if and only if for every $u, v \in D$ there exists $j \in J(u-v)$ such that

$$
\langle T(u)-T(v), j\rangle \leq\|u-v\|^{2}
$$

where $J: X \rightarrow 2^{X^{*}}$ is the normalized duality mapping which is defined by

$$
J(u)=\left\{j \in X^{*}:\langle u, j\rangle=\|u\|^{2},\|j\|=\|u\|\right\}
$$

(see Browder [4] and Kato [12]). The symbol $\langle\cdot, \cdot\rangle$ denotes the generalized duality pairing. It is an immediate consequence of the Hahn-Banach Theorem that $J(u)$ is nonempty for each $u$ in $X$. It is also known that $J(u)$ is single-valued if and only if $X$ is smooth. By the way, this latter notion means that

$$
\lim _{t \rightarrow 0} \frac{\|x+t h\|-\|x\|}{t}
$$

Received by the editors September 18, 1998 and, in revised form, January 22, 1999.

1991 Mathematics Subject Classification. Primary 47H10.

Key words and phrases. Pseudo-contractive mappings, uniformly Gâteaux differentiable norm.

This paper was carried out while the second author was visiting the University of Alabama in Huntsville under the financial support of the LG Yonam Foundation, 1998, and he would like to thank Professor Claudio H. Morales for his hospitality in the Department of Mathematics. 
exists for each $x$ and $h$ on the unit sphere $U$ of $X$. When this is the case, we say that the norm of $X$ is Gâteaux differentiable. Moreover, if for each $h$ in $U$ the limit defined by (2) is uniformly attained for $x$ in $U$, we say that the norm of $X$ is uniformly Gâteaux differentiable. It is also known that if $X$ has a uniformly Gâteaux differentiable norm, then the mapping $J$ is uniformly continuous on bounded sets from the norm topology on $X$ to the weak* topology on $X^{*}$.

In addition to generalizing the nonexpansive mappings (mappings $T: D \rightarrow X$ for which $\|T(x)-T(y)\| \leq\|x-y\|$ for all $x$ and $y$ in $D$ ), the pseudo-contractive ones are characterized by the fact that $T$ is pseudo-contractive if, and only if, $I-T$ is accretive (see [4 12]). Continuous interest in mapping theory for accretive operators, particularly as it relates to existence theorems for nonlinear ordinary and partial differential equations, has prompted a corresponding interest in fixed point theory for pseudo-contractive mappings (see, e.g., [4], 6], [8], 12], [13, [15]).

The purpose of this paper is to continue the discussion concerning the strong convergence of the path $t \rightarrow x_{t}, 0 \leq t<1$, defined by (3) below. We obtain further extensions of various results obtained quite recently by Jung-Kim [11, Morales 17], Takahashi-Kim [21, and $\mathrm{Xu}$ [22] to more general types of spaces, as well as families of operators. It should be mentioned that the study of this type of problem began over thirty years ago. To the best of our knowledge, the first results of this nature were obtained by Browder [3] and Halpern [10] for nonexpansive selfmappings defined on Hilbert spaces. In the years to follow, several extensions to more general Banach spaces and/or nonexpansive nonself-mappings were obtained, including some local versions for this very same type of operator, which can be found in [20], [14], [5], 17] among others. In fact, Reich suggested, for nonexpansive selfmappings, that the convergence of the path would hold for more general types of spaces (see Remark 1 of [20]).

It is relevant to the first two theorems of this paper to note that while every uniformly smooth Banach space is a reflexive Banach space with a uniformly Gâteaux differentiable norm, the converse does not hold. To see this, consider $X$ to be the direct sum $l^{2}\left(l^{p_{n}}\right)$, the class of all those sequences $x=\left\{x_{n}\right\}$ with $x_{n} \in l^{p_{n}}$ and $\|x\|=\left(\sum_{n<\infty}\left\|x_{n}\right\|^{2}\right)^{1 / 2}$ (see [7]). Now, if $1<p_{n}<\infty$ for all $n \in \mathbb{N}$, where either $\lim \sup _{n \rightarrow \infty} p_{n}=\infty$ or $\liminf _{n \rightarrow \infty} p_{n}=1$, then $X$ is a reflexive Banach space (see 7]) with a uniformly Gâteaux differentiable norm (see [23]), but is not uniformly smooth (see [7]). We also observe that spaces which enjoy the fixed point property (F.P.P.) for nonexpansive self-mappings are not necessarily spaces with a uniformly Gâteaux differentiable norm. On the other hand, the converse of this fact appears to be unknown as well.

\section{Preliminaries}

Throughout the paper we will use the following notion. Let $X$ be a linear space with $K \subset X$. For $x \in K$, the inward set $I_{K}(x)$ is defined by

$$
I_{K}(x)=\{x+\lambda(u-x): u \in K, \lambda \geq 1\} .
$$

A mapping $T: K \rightarrow X$ is said to satisfy the weakly inward condition if

$$
T x \in \operatorname{cl}\left[I_{K}(x)\right] \quad \text { for all } x \in K .
$$

On the other hand, if $T x \in I_{K}(x)$ for each $x \in K, T$ is said to, simply, satisfy the inward condition. 
Proposition 1. Let $X$ be a Banach space. Suppose $K$ is a closed convex subset of $X$ and $T: K \rightarrow X$ a continuous pseudo-contractive mapping satisfying the weakly inward condition. Then for each $x_{0} \in K$, there exists a unique path $t \rightarrow x_{t} \in K$, $t \in[0,1)$, satisfying

$$
x_{t}=t T x_{t}+(1-t) x_{0} .
$$

Proof. For each $t \in[0,1)$, the mapping $T_{t}(x)=t T x+(1-t) x_{0}$ is continuous and strongly pseudo-contractive which satisfies the weakly inward condition. Therefore by Corollary 1 of $[8], T_{t}$ has a unique fixed point $x_{t}$ in $K$. This means the equation

$$
x_{t}=t T x_{t}+(1-t) x_{0}
$$

has a unique solution for each $t \in[0,1)$. To see the continuity we follow the argument given in [17]. Let $t, t_{0} \in[0,1)$. Then there exists $j \in J\left(x_{t}-x_{t_{0}}\right)$, such that

$$
\left\langle x_{t}-x_{t_{0}}, j\right\rangle=\left\langle\left(t-t_{0}\right)\left(T x_{t_{0}}-x_{0}\right), j\right\rangle+t\left\langle T x_{t}-T x_{t_{0}}, j\right\rangle,
$$

and this implies that

$$
\left\|x_{t}-x_{t_{0}}\right\|^{2} \leq\left\|x_{0}-T x_{t_{0}}\right\|\left\|x_{t}-x_{t_{0}}\right\|\left|t-t_{0}\right|+t\left\|x_{t}-x_{t_{0}}\right\|^{2} .
$$

Therefore

$$
\left\|x_{t}-x_{t_{0}}\right\| \leq\left[\left\|x_{0}-T x_{t_{0}}\right\| /(1-t)\right]\left|t-t_{0}\right| .
$$

This completes the proof.

Proposition 2. Let $X$ be a Banach space and let $K$ be a closed convex subset of $X$. Suppose $T: K \rightarrow X$ is a pseudo-contractive mapping such that for each $z \in K$, the equation

$$
x=t T x+(1-t) z
$$

has a solution $z_{t}$ for every $t \in(0,1)$. Then:

(i) (cf. Lemma 2(ii) of [16]). The function $h(t)=\left\|z_{t}-T z_{t}\right\|$ is monotone decreasing on $[0,1)$, and in particular $\left\|z_{t}-T z_{t}\right\| \leq\|z-T z\|$.

(ii) If $\left\{w_{t}\right\}$ is the path corresponding to the initial point $w$, we have

$$
\left\|z_{t}-w_{t}\right\| \leq\|z-w\|
$$

(iii) If for some $u \in K$, the path $\left\{u_{t}\right\}$ with $u_{t}=t T u_{t}+(1-t) u$ is bounded, then any other path $\left\{z_{t}\right\}$ with $z_{t}=t T z_{t}+(1-t) z$, where $z$ is arbitrarily chosen in $K$, is also bounded.

(iv) If $T$ has a fixed point in $K$, then the path $\left\{z_{t}\right\}$ is also bounded.

(v) If $v$ is a fixed point of $T$, there exists $j \in J\left(z_{t}-v\right)$ such that

$$
\left\langle z_{t}-z, j\right\rangle \leq 0 \text {. }
$$

Proof. (i) Let $0 \leq s \leq t<1$. Then

$$
\begin{aligned}
\left\|z_{t}-T z_{t}\right\| & =\frac{1-t}{t}\left\|z_{t}-z\right\| \\
& \leq \frac{1-t}{t}\left[\left\|z_{t}-z_{s}\right\|+\left\|z_{s}-z\right\|\right] \\
& \leq \frac{1-t}{t}\left[\frac{t-s}{1-t}+\frac{s}{1-s}\right]\left\|z_{s}-T z_{s}\right\| \\
& \leq\left\|z_{s}-T z_{s}\right\| .
\end{aligned}
$$


(ii) Since $T$ is pseudo-contractive, for each $t \in[0,1)$ there exists $j \in J\left(z_{t}-w_{t}\right)$ such that

$$
\begin{aligned}
\left\langle z_{t}-w_{t}, j\right\rangle & =t\left\langle T z_{t}-T w_{t}, j\right\rangle+(1-t)\langle z-w, j\rangle \\
& \leq t\left\|z_{t}-w_{t}\right\|^{2}+(1-t)\|z-w\|\left\|z_{t}-w_{t}\right\|
\end{aligned}
$$

Therefore $\left\|z_{t}-w_{t}\right\| \leq\|z-w\|$.

(iii) The proof follows from part (ii).

(iv) Let $v$ be a fixed point of $T$. Then by the pseudo-contractiveness, there exists $j \in J\left(z_{t}-v\right)$ such that

$$
\begin{aligned}
\left\langle z_{t}-v, j\right\rangle & =\left\langle t T z_{t}+(1-t) z-v, j\right\rangle \\
& =t\left\langle T z_{t}-T v, j\right\rangle+(1-t)\langle z-v, j\rangle \\
& \leq t\left\langle z_{t}-v, j\right\rangle+(1-t)\|z-v\| .
\end{aligned}
$$

Therefore $\left\|z_{t}-v\right\| \leq\|z-v\|$, which implies that $\left\{z_{t}\right\}$ is bounded.

(v) Let $v$ be a fixed point of $T$. Then by the pseudo-contractiveness, there exists $j \in J\left(z_{t}-v\right)$ such that

$$
\begin{aligned}
\left\langle z_{t}-z, j\right\rangle & =t\left\langle T z_{t}-z, j\right\rangle \\
& =t\left\langle T z_{t}-T v, j\right\rangle+t\langle v-z, j\rangle \\
& \leq t\left\langle z_{t}-z, j\right\rangle .
\end{aligned}
$$

Therefore $\left\langle z_{t}-z, j\right\rangle \leq 0$.

Next we state a rather simple inequality that most recently has been referred to as a new result, although the result has been known for about thirty years. Indeed, this is Lemma 1 of Petryshyn [18; even earlier, Asplund 11 proved a general result for single-valued duality mappings that can be used to derive this lemma. We include its proof for the sake of completeness.

Lemma 1. Let $X$ be a normed space. For each $x, y \in X$ and $j \in J(x+y)$ we have

$$
\|x+y\|^{2} \leq\|x\|^{2}+2\langle y, j\rangle \text {. }
$$

Proof. Since $j \in J(x+y)$, we have

$$
\begin{aligned}
\|x+y\|^{2} & =\langle x+y, j\rangle \\
& =\langle x, j\rangle+\langle y, j\rangle \\
& \leq \frac{1}{2}\left(\|x\|^{2}+\|j\|^{2}\right)+\langle y, j\rangle .
\end{aligned}
$$

Therefore the conclusion follows.

\section{Main Results}

Theorem 1. Let $X$ be a Banach space with a uniformly Gâteaux differentiable norm. Suppose $K$ is a closed convex subset of $X$ and $T: K \rightarrow X$ a continuous pseudocontractive mapping satisfying the weakly inward condition. Suppose every closed bounded convex subset of $K$ has the F.P.P. for nonexpansive self-mappings. If there exists $u_{0} \in K$ such that the set

$$
E=\left\{x \in K: T(x)=\lambda x+(1-\lambda) u_{0} \text { for some } \lambda>1\right\}
$$

is bounded, then the path $\left\{x_{t}: t \in[0,1)\right\}$ described by (3) converges strongly to a fixed point of $T$. 
Proof. Due to Proposition 1, it just remains to show the strong convergence of the path $\left\{x_{t}\right\}$ as $t \rightarrow 1^{-}$. We first observe that the mapping $f(x)=2 x-T(x)$ has a nonexpansive inverse, denoted by $g$, which maps $K$ into $K$ as a consequence of Theorem 6 of [15]. We now let $x_{n}=x_{t_{n}}$ for $t_{n} \rightarrow 1^{-}$as $n \rightarrow \infty$. Since $E$ is bounded, so is $\left\{T x_{n}\right\}$, and thus $x_{n}-T x_{n} \rightarrow 0$ as $n \rightarrow \infty$. Consequently $x_{n}-g\left(x_{n}\right) \rightarrow 0$. Since $\left\{x_{n}\right\}$ is a bounded sequence, let $L=\limsup _{n \rightarrow \infty}\left\|x_{n}\right\|$. Then define the set $K_{0}$ by

$$
K_{0}=\left\{u \in K: \limsup _{n \rightarrow \infty}\left\|x_{n}-u\right\| \leq L\right\} .
$$

Clearly $K_{0}$ is nonempty since $0 \in K_{0}$. In addition $K_{0}$ is closed bounded and convex, and since

$$
\left\|g(u)-x_{n}\right\| \leq\left\|u-x_{n}\right\|+\left\|x_{n}-g\left(x_{n}\right)\right\|,
$$

$K_{0}$ is also invariant under $g$. And therefore, due to the assumption on $K, g$ has a fixed point in $K_{0}$. Denote such a fixed point by $v$. Since $T$ is pseudo-contractive and $v$ is a fixed point of $T$, we may derive from Proposition 2(v) that

$$
\left\langle x_{n}-x_{0}, J\left(x_{n}-v\right)\right\rangle \leq 0
$$

for all $n \in \mathbb{N}$. Now let $x=x_{0}-v$ and $t \in(0,1]$. Then by Lemma 1 we have

$$
\left\|x_{n}-v-t x\right\|^{2} \leq\left\|x_{n}-v\right\|^{2}+2\left\langle-t x, J\left(x_{n}-v-t x\right)\right\rangle .
$$

Let $\varepsilon>0$. Then by assumption there exists $\delta>0$ such that

$$
\left\langle x, J\left(x_{n}-v\right)\right\rangle<\varepsilon+\left\langle x, J\left(x_{n}-v-t x\right)\right\rangle
$$

for all $t \in(0, \delta)$. Consequently,

$$
\left\langle x, J\left(x_{n}-v\right)\right\rangle<\varepsilon+\frac{1}{2 t}\left[\left\|x_{n}-v\right\|^{2}-\left\|x_{n}-v-t x\right\|^{2}\right] .
$$

Then we may choose a subsequence $\left\{x_{n_{k}}\right\}$ of $\left\{x_{n}\right\}$ such that

$$
\limsup _{k \rightarrow \infty}\left\langle x, J\left(x_{n_{k}}-v\right)\right\rangle \leq 0 .
$$

Combining (6) and (17), we conclude that $\left\{x_{n_{k}}\right\}$ converges strongly to $v$. To complete the proof, let $\left\{x_{m_{k}}\right\}$ be another subsequence of $\left\{x_{t}: t \in[0,1)\right\}$ such that $x_{m_{k}}=x_{t_{m_{k}}}, t_{m_{k}} \rightarrow 1^{-}$as $k \rightarrow \infty$, and $x_{m_{k}} \rightarrow w$, where $T w=w$. Then Proposition 2(v) implies that $\left\langle v-x_{0}, J(v-w)\right\rangle \leq 0$ and $\left\langle w-x_{0}, J(w-v)\right\rangle \leq 0$. Hence $v=w$ and the strong $\lim _{t \rightarrow 1^{-}} x_{t}$ exists, which completes the proof.

We remark that Theorem 1 generalizes several recent results of this nature. Particularly, it extends Theorem 1] of [21], where, in addition to the reflexivity assumption, it is also assumed that the operator $T$ is nonexpansive and maps $K$ into itself. Perhaps we should point out that the use of the classical theorem of Martin [15] has been vital in extending many results for nonexpansive mappings to pseudo-contractive ones.

Corollary 1. Let $X$ be a reflexive Banach space with a uniformly Gâteaux differentiable norm. Suppose $K$ is a closed convex subset of $X$ with normal structure, and $T: K \rightarrow X$ a continuous pseudo-contractive mapping satisfying the weakly inward condition. If the set $E$ described by (5) is bounded, then the path $\left\{x_{t}\right\}$ converges strongly to a fixed point of $T$ as $t \rightarrow 1^{-}$. 
Corollary 2. Let $X$ be a uniformly convex Banach space with a uniformly Gâteaux differentiable norm. Suppose $K$ is a closed convex subset of $X$, and $T: K \rightarrow X$ a continuous pseudo-contractive mapping satisfying the weakly inward condition. If the set $E$ described by (5) is bounded, then the path $\left\{x_{t}\right\}$ converges strongly to a fixed point of $T$ as $t \rightarrow 1^{-}$.

We should observe that Corollary 2 is a significant extension of Theorem 2 of [1], which is done for nonexpansive mappings under the additional assumption that $K$ is a sunny nonexpansive retract of $X$.

As we well know, a Banach space $X$ is uniformly smooth if and only if $X^{*}$ is uniformly convex. This means Theorem 1 is also an extension of Theorem 5 of [17, as the following corollary shows.

Corollary 3. Let $X^{*}$ be uniformly convex and let $K$ and $T$ be as in Theorem 1 . Suppose $T$ satisfies the weakly inward condition. Then the path $t \rightarrow x_{t}, t \in[0,1)$, strongly converges to a fixed point of $T$ provided that $E$, defined by (5), is bounded.

Corollary 4. Let $X$ be a uniformly smooth Banach space and let $K$ be a closed convex subset of $X$, and $T: K \rightarrow X$ a continuous pseudo-contractive mapping satisfying the weakly inward condition. If $T$ has a fixed point, then the path $\left\{x_{t}\right\}$ converges strongly to a fixed point of $T$ as $t \rightarrow 1^{-}$.

Proof. Without loss of generality, we may assume that $0 \in K$. Let $v$ be a fixed point of $T$ and let $T x=\lambda x$ for some $\lambda>1$. Then there exists $j \in J(x-v)$ such that

$$
\langle\lambda x-v, j\rangle \leq\|x-v\|^{2} .
$$

This implies that $\|x-v\| \leq\|v\|$. Therefore the set $E$ is bounded. The rest of the proof follows from Corollary 3 .

As we observed in Proposition 2(iv), any path $\left\{x_{t}\right\}$ with initial point $x_{0}$ is bounded if $T$ is an arbitrary pseudo-contractive mapping with a fixed point in $K$. Therefore as a consequence of Theorem 1 we can derive the following result which happens to be an extension of Theorem 1 of [22], where the inwardness condition on $T$ is actually assumed.

Corollary 5. Let $X$ be a uniformly smooth Banach space and let $K$ be a closed convex subset of $X$ and $T: K \rightarrow X$ a nonexpansive mapping with a fixed point in $K$. If $T$ satisfies the weakly inward condition, then the path $\left\{x_{t}\right\}$ converges strongly to a fixed point of $T$ as $t \rightarrow 1^{-}$.

Next we substitute the Fixed Point Property assumption, mentioned in Theorem 1 by assuming that the space $X$ is reflexive and strictly convex.

Theorem 2. Let $X$ be a reflexive and strictly convex Banach space with a uniformly Gatteaux differentiable norm. Suppose $K$ is a closed convex subset of $X$ and $T: K \rightarrow X$ a continuous pseudo-contractive mapping with a fixed point in $K$. If $T$ satisfies the weakly inward condition, then the path $\left\{x_{t}\right\}$ strongly converges to a fixed point of $T$ as $t \rightarrow 1^{-}$.

Proof. Let $v$ be a fixed point of $T$. Since $\left\|x_{t}-v\right\| \leq\left\|x_{0}-v\right\|$ for all $t \in[0,1)$, we have that $\left\{x_{t}\right\}$ is bounded. Let $g$ be the nonexpansive mapping introduced in the proof of Theorem 1. Let $x_{n}=x_{t_{n}}$ while $t_{n} \rightarrow 1^{-}$as $n \rightarrow \infty$. Then $x_{n}-g\left(x_{n}\right) \rightarrow 0$. 
We now define $h: X \rightarrow \mathbb{R}^{+}$by $h(x)=\lim \sup _{n \rightarrow \infty}\left\|x_{n}-x\right\|$. Then $h$ is continuous and convex, and therefore the set

$$
K_{0}=\{u \in K: h(u)=\inf \{h(x): x \in K\}\}
$$

is a nonempty closed bounded convex subset of $K$, which is also invariant under $g$. To be able to use the argument of the proof of Theorem 1, we just need to show that the set $K_{0}$ contains a fixed point of $g$. Since the fixed points of $T$ are also fixed points of $g$, let $v$ be one of those, and define the function $f: K_{0} \rightarrow \mathbb{R}$ by $f(u)=\|u-v\|$. Then by Theorem 1.2 of [2] p. 79] we conclude that the set

$$
C=\left\{u \in K_{0}: f(u)=\inf \left\{f(y): y \in K_{0}\right\}\right\}
$$

is nonempty, and since $X$ is strictly convex, $C=\left\{u_{0}\right\}$ for some $u_{0} \in K_{0}$. We also know that $g(v)=v$ and

$$
\left\|g\left(u_{0}\right)-v\right\|=\left\|g\left(u_{0}\right)-g(v)\right\| \leq\left\|u_{0}-v\right\| .
$$

Therefore $g\left(u_{0}\right)=u_{0}$. We now follow the proof of Theorem 1 .

We remark that Theorem 2 appears to be independent of Theorem[1. On the one hand, it is easy to find examples of spaces which satisfy the F.P.P. for nonexpansive mappings, which are not strictly convex. However, it appears to be unknown whether a reflexive and strictly convex space satisfies the F.P.P. for nonexpansive mappings.

As a consequence of Theorem 2, we may derive the following result.

Corollary 6. Let $X$ and $K$ be as in Theorem 2 and let $T: K \rightarrow X$ be a continuous pseudo-contractive mapping satisfying the weakly inward condition. Then the path $\left\{x_{t}\right\}$ converges strongly to a fixed point of $T$ as $t \rightarrow 1^{-}$if $T$ satisfies the following conditions:

(i) If $\left\{u_{n}\right\}$ is any sequence in $K$ such that $u_{n}-T u_{n} \rightarrow 0$ as $n \rightarrow \infty$, then there exists $u \in K$ with $u-T u=0$.

(ii) Either $\|x-T x\| \rightarrow \infty$ as $\|x\| \rightarrow \infty$ or $\langle x-T x, J(x)\rangle \geq 0$ for $\|x\| \geq R$ and some $R>0$.

Proof. We know from Proposition 2(i) that

$$
\left\|x_{t}-T x_{t}\right\| \leq\left\|x_{0}-T x_{0}\right\| \quad \text { for } t \in[0,1),
$$

and hence, by the first assumption in (ii), we obtain that $\left\{x_{t}\right\}$ is bounded. In case of the second assumption of (ii), we prove that the set $E$, defined earlier, is bounded, and hence, Proposition 2(iii) implies that $\left\{x_{t}\right\}$ is also bounded. Consequently $\left\|x_{t}-T x_{t}\right\| \rightarrow 0$ as $t \rightarrow 1^{-}$. Therefore (i) implies that $T$ has a fixed point. The remainder of the proof follows from Theorem 2

We next obtain a convergence of the path described by (3) for spaces admitting a weakly sequentially continuous duality mapping. In this case, the convexity assumption on the domain of the operator $T$ can be dropped, while a weaker continuity assumption on the operator itself can be assumed.

Theorem 3. Let $X$ be a reflexive Banach space which admits a weakly sequentially continuous duality mapping from $X$ to $X^{*}$. Suppose $K$ is a closed subset of $X$ and $T: K \rightarrow X$ a demicontinuous pseudo-contractive mapping such that for some $x_{0} \in K$, the equation

$$
x=t T x+(1-t) x_{0}
$$


has a solution $x_{t}$ in $K$ for each $t \in[0,1)$. If the path $\left\{x_{t}\right\}$ is bounded, then it converges strongly to a fixed point of $T$ as $t \rightarrow 1^{-}$.

Proof. We first observe that due to Lemma 1 of $[9$, in this case the duality mapping $J$ is single-valued. Now suppose $x_{n}=x_{t_{n}}$ with $t_{n} \rightarrow 1^{-}$a weakly convergent sequence, say to $x$. Then from (8) we get

$$
\left\langle\left(t_{n}^{-1}-1\right) x_{n}-\left(t_{m}^{-1}-1\right) x_{m}, J\left(x_{n}-x_{m}\right)\right\rangle \leq\left(t_{n}^{-1}-t_{m}^{-1}\right)\left\langle x_{0}, J\left(x_{n}-x_{m}\right)\right\rangle .
$$

By letting $m \rightarrow \infty$, we obtain

$$
\left\langle x_{n}, J\left(x_{n}-x\right)\right\rangle \leq\left\langle x_{0}, J\left(x_{n}-x\right)\right\rangle
$$

and thus

$$
\left\|x_{n}-x\right\|^{2} \leq\left\langle x_{0}-x, J\left(x_{n}-x\right)\right\rangle .
$$

Therefore $x_{n} \rightarrow x$ as $n \rightarrow \infty$. We now follow the argument given in the proof of Theorem 1 to conclude that $\left\{x_{t}\right\}$ converges strongly to $x$ in $K$. The demicontinuity completes the proof.

We should remark that Theorem 3 appears to be a new result, since it is not known whether a reflexive Banach space admitting a weakly sequentially continuous duality mapping has a uniformly Gâteaux differentiable norm. However, it is known that they enjoy the F.P.P. for nonexpansive mappings.

Corollary 7. Let $X$ be as in Theorem 9 and let $K$ be a closed convex subset of $X$. Suppose $T: K \rightarrow X$ is a continuous pseudo-contractive mapping satisfying the weakly inward condition. If the set $E$ defined by (5) is bounded, then the path $\left.\left\{x_{t}: t \in[0,1]\right)\right\}$ is relatively compact in the strong topology of $X$.

Proof. By Proposition [1, the path described by (8) is well defined in $K$. Since $E$ is bounded, so is the path, and the conclusion follows from Theorem 3

Corollary 7 is a generalization of Theorem 2 of Browder [3], who assumes in addition that $X$ is uniformly convex and that $T$ is a nonexpansive mapping defined in the whole space $X$.

Finally, as a consequence of the proofs of Theorem 1 and Corollary 6 , we obtain the following fixed point theorem, which is an extension of Proposition 4 of Martin [15].

Theorem 4. Let $X$ be a Banach space with a uniformly Gâteaux differentiable norm. Suppose $K$ is a closed convex subset of $X$ and $T: K \rightarrow X$ a continuous pseudo-contractive mapping satisfying the weakly inward condition. Suppose every closed bounded convex subset of $K$ has the F.P.P. for nonexpansive self-mappings. If $I-T$ is unbounded on unbounded subsets of $K$, then $T$ has a fixed point in $K$.

We observe that Theorem 4 is part of Theorem 5 of Reich [19; however his proof implicitly uses that $T$ must be a bounded operator.

\section{ACKNOWLEDGMENT}

The authors wish to thank the referee for the valuable suggestions to improve the writing of this paper. 


\section{REFERENCES}

1. E. Asplund, Positivity of duality mappings, Bull. Amer. Math. Soc. 73 (1967), 200-203. MR 34:6481

2. V. Barbu and Th. Precupanu, Convexity and Optimization in Banach spaces, Editura Academiei R. S. R., Bucharest, 1978. MR 58:23939

3. F. E. Browder, Convergence of approximants to fixed points of nonexpansive nonlinear mappings in Banach spaces, Archs. Ration. Mech. Anal. 24 (1967), 82-90. MR 34:6582

4. F. E. Browder, Nonlinear mappings of nonexpansive and accretive type in Banach spaces, Bull. Amer. Math. Soc. 73 (1967), 875-882. MR 38:581

5. R. E. Bruck, W. A. Kirk and S. Reich, Strong and weak convergence theorems for locally nonexpansive mappings in Banach spaces, Nonlinear Anal. Theory Methods Appl. 6 (1982), 151-155. MR 83j:47040

6. M. G. Crandall, Differential equations on convex sets, J. Math. Soc. Japan 22 (1970), 443-455. MR 42:3388

7. M. M. Day, Reflexive Banach spaces not isomorphic to uniformly convex spaces, Bull. Amer. Math. Soc. 47 (1941), 313-317. MR 2:221b

8. K. Deimling, Zeros of accretive operators, Manuscripta Math. 13 (1974), 365-374. MR 50:3030

9. J.-P. Gossez and E. Lami Dozo, Some geometric properties related to the fixed point theory for nonexpansive mappings, Pacific J. Math. 40 (1972), 565-573. MR 46:9815

10. B. Halpern, Fixed points of nonexpanding maps, Bull. Amer. Math. Soc. 73 (1967), 957-961. MR 36:2022

11. J. S. Jung and S. S. Kim, Strong convergence theorems for nonexpansive nonself-mappings in Banach spaces, Nonlinear Anal. Theory Methods Appl. 33 (1998), 321-329. MR 99h:47068

12. T. Kato, Nonlinear semigroups and evolution equations, J. Math. Soc. Japan 19 (1967), 508-520. MR 37:1820

13. W. A. Kirk, A fixed point theorem for local pseudo-contractions in uniformly convex spaces, Manuscripta Math. 30 (1979), 98-102. MR 80k:47064

14. W. A. Kirk and C. H. Morales, On the approximation of fixed points of locally nonexpansive mappings, Canad. Math. Bull. 24 (1981), 441-445. MR 83h:47040

15. R. H. Martin, Differential equations on closed subsets of a Banach space, Trans. Amer. Math. Soc. 179 (1973), 399-414. MR 47:7537

16. C. H. Morales, On the fixed-point theory for local k-pseudocontractions, Proc. Amer. Math. Soc. 81 (1981), 71-74. MR 82c: 47072

17. C. H. Morales, Strong convergence theorems for pseudo-contractive mappings in Banach space, Houston J. Math. 16 (1990), 549-557. MR 92f:47063

18. W. V. Petryshyn, A characterization of strict convexity of Banach spaces and other uses of duality mappings, J. Funct. Anal. 6 (1970), 282-291. MR 44:4496

19. S. Reich, Iterative methods for accretive sets, Proc. Conf. on Nonlinear Equations in Abstract Spaces, Acad. Press (1978), 317-326. MR 82c:47065

20. S. Reich, Strong convergence theorems for resolvents of accretive operators in Banach spaces, J. Math. Anal. Appl. 75 (1980), 287-292. MR 82a:47050

21. W. Takahashi and G. E. Kim, Strong convergence of approximants to fixed points of nonexpansive nonself-mappings in Banach spaces, Nonlinear Anal. Theory Methods Appl. 32 (1998), 447-454. MR 99h:47063

22. H. K. Xu, Approximating curves of nonexpansive nonself-mappings in Banach spaces, C. R. Acad. Sci. Paris Sér. I. Math. 325 (1997), 151-156. MR 98i:47052

23. V. Zizler, On some rotundity and smoothness properties of Banach spaces, Dissert. Math. 87 (1971), 5-33. MR 45:9108

Department of Mathematics, University of Alabama, Huntsville, Alabama 35899

E-mail address: morales@math.uah.edu

Department of Mathematics, Dong-A University, Pusan 604-714, Korea

E-mail address: jungjs@mail.donga.ac.kr 